\title{
Modeling of an Elastic Joint: An Experimental Setup Approach
}

\author{
Vítor H. Pinto ${ }^{1,3(凶)}$, José Lima ${ }^{2}$, José Gonçalves ${ }^{2}$, and Paulo Costa ${ }^{1,3}$ \\ 1 Faculty of Engineering, FEUP - University of Porto, Porto, Portugal \\ \{vitorpinto,paco\}@fe.up.pt \\ 2 IPB - Polytechnic Institute of Bragança, Bragança, Portugal \\ \{jllima, goncalves\}@ipb.pt \\ 3 CRIIS - Centre for Robotics in Industry and Intelligent Systems, \\ INESC TEC - Institute for Systems and Computer Engineering, \\ Technology and Science, Porto, Portugal
}

\begin{abstract}
Throughout this paper it is presented a novel elastic joint configuration, being compared with other similar joints found in recent literature. It is presented its modeling, being its estimation process developed offline, based on a proposed experimental setup. This setup enables to monitor and collect data from an absolute encoder and a load cell. Some data obtained from these sensors is then graphically represented, like angle and torque, obtaining some parameters. Finally, through an optimization process, where the error of the angle is minimized, the remaining parameters of the joint are estimated, thus obtaining a realistic model of the system.
\end{abstract}

Keywords: Elastic joint $\cdot$ Modeling $\cdot$ Experimental setup $\cdot$ Parameter estimation - Optimization

\section{Introduction}

To increase safety and robustness in the performance of industrial robots, which otherwise could easily break their joints, or hurt someone, the use of elastic joints is increasingly found in the literature and in the industry. These mechanisms contain elastic components (such as springs), which introduce a mechanical energy storage in the system, which can increase the maximum output power [1]. In [2], Giusti et al. have designed and implemented successfully a controller based on the analysis of the inverse dynamics of the elastic joint, and applied a structured controller. The testbed mixes rigid and elastic joints on an interesting configuration. The usage of elastic joints for exoskeletons is also expanding, possibly due to the decrease in the size of the actuators. An example is the work is presented in [3] for a variable viscoelastic joint system. Another interesting application example for elastic joints are deployable mechanisms, as the one presented by

(C) The Editor(s) (if applicable) and The Author(s), under exclusive license to Springer Nature Switzerland AG 2021

J. A. Gonçalves et al. (Eds.): CONTROLO 2020, LNEE 695, pp. 676-685, 2021. https://doi.org/10.1007/978-3-030-58653-9_65 
Zhang et al. [4], where the singularities of a system like the presented are analyzed and successfully avoided. The Quasi-Direct Drive is also an example of the technology applied to actuate an elastic joint, as presented in [5] for a quadruped.

As it can be easily understood, all the mentioned research has a same primary step the modeling phase, applied to translate mathematically the behavior of the system. This is required to be able to design suitable controllers, test them in simulation and obtain relevant results. Thus, the closer to reality the developed model is, the better designed the controller can be in theory, and be applied in practice. However, all the robotic systems mentioned above include components that adds uncertainties and non-linearities to it, making it more complex to model.

Estimation techniques can be applied to help modeling the system, since these can be more suited to the obtained solution. The techniques applied can be diverse, from evolutionary algorithms, such as Montazeri et al. use in [6], recursive methods as Gonzalez et al. [7] use for their third-order modeling, through the use of neural networks, as explained in [8] or even experimentally, as the authors of [9] and [10] present.

Considering that the essential characteristics of the elastic joint developed for this work are not expected to suffer structural changes, the estimation of its dynamic parameters will be carried out previously, with offline techniques, and using experiments that allow to obtain them.

This paper presents a novel approach to an elastic joint and the process of modeling it is described. An introduction to the topic was performed in Sect.1, and the proposed model will be introduced throughout Sect.2. Section 3 will state the obtained results by the carried out experiments, and in Sect. 4 there are presented the conclusions and future work.

\section{Proposed Model}

Throughout this section, the model for the elastic joint will be presented. Some hardware tests were performed to estimate its parameters, and these will be further detailed.

For the elastic joint construction, a passive system was developed. Applying 4 springs, attached to a circular support part as represented in Fig. 1, a rotational force is applied in the support's centre of rotation, is passed to the next link through the springs, providing elasticity and cushioning to the rotational movement. To connect all the parts, as can be noticeable by the mentioned figure, bearings are used to decrease the rotational friction, as well as to allow a tighter connection between all the parts.

The mathematical/physical representation of the system, with its respective constants, velocities and torques is present in Fig. 2.

Given the proposed elastic joint, its model will become as follow:

$$
\begin{aligned}
\tau_{K} & =K \cdot\left(\theta_{m}-\theta_{j}\right) \\
\tau_{B} & =B \cdot\left(\dot{\theta}_{m}-\dot{\theta}_{j}\right)
\end{aligned}
$$




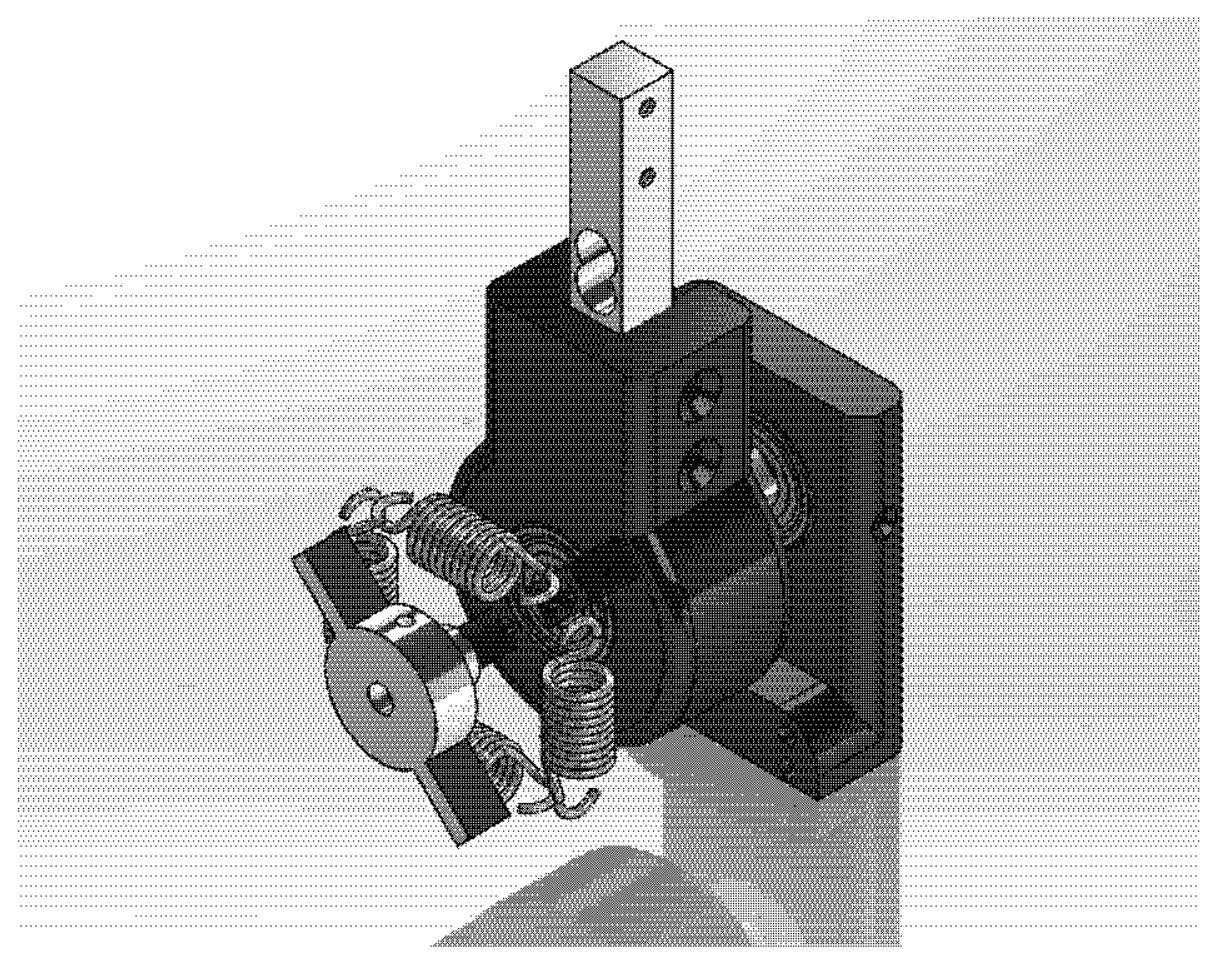

Fig. 1. Simulated representation of the elastic joint

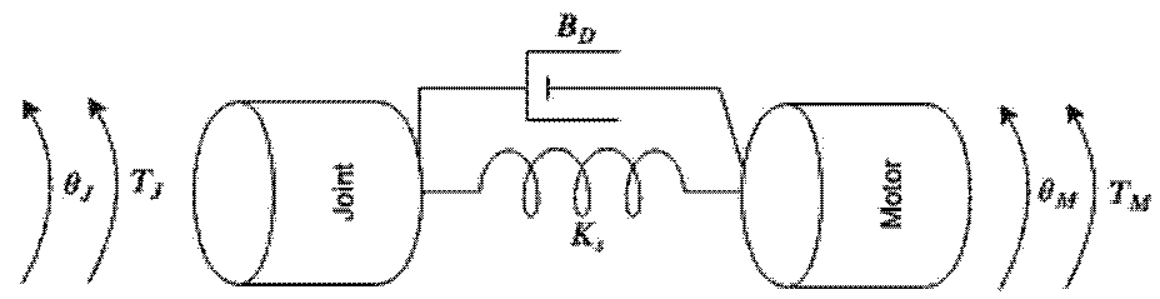

Fig. 2. Motor joint representation

$$
\begin{gathered}
J \cdot \ddot{\theta}=\sum \tau=\tau_{K}+\tau_{B} \\
\frac{d \omega}{d t}=\frac{1}{J}(-K \theta-B \dot{\omega}) \\
J \cdot \frac{d^{2} \theta(t)}{d t}+B \cdot \frac{d \theta(t)}{d t}+K \cdot \theta(t)=0 \\
J \cdot\left(s^{2} \theta(s)-s \theta(t=0)-\frac{d \theta}{d t}(t=0)\right)+B \cdot(s \theta(s)-\theta(t=0))+K \cdot \theta(s)=0
\end{gathered}
$$


Considering that the initial conditions can be not null, the following variables take values:

$$
\begin{gathered}
\theta(t=0)=\theta_{0} \\
\frac{d \theta(t=0)}{d t}=\omega(t=0)=0 \\
J s^{2} \theta(s)-J s \theta_{0}+B s \theta(s)-B \theta_{0}+K \theta(s)=0 \\
\theta(s)=\frac{\theta_{0} \cdot(J s+B)}{J s^{2}+B s+K} \\
\theta(s)=\theta_{0} \cdot \frac{\left(s+\frac{B}{J}\right)}{s^{2}+\left(\frac{B}{J}\right) s+\left(\frac{K}{J}\right)}
\end{gathered}
$$

Similar to what was described in the above subsection, for modeling the elastic joint, as well as for estimating the parameters associated with it, some hardware tests will also be performed, in order to obtain parameters $J, K$ and $B$, consisting of:

- Pushing/Pulling the link attached to the elastic joint until a certain angle;

- Pushing/Pulling the link attached to the elastic joint and releasing, allowing it to stabilize;

To conduct these tests, it is needed to know the joint's angular position, as well as the applied torque. So, to obtain the exact angular displacement, an absolute encoder was placed exactly concentrically with the axis of rotation of the joint. Also, to obtain the torque, a load cell was placed in the center of the link to evaluate the exerted force which can, using the distance to the rotational axis, be traduced to the torque. Figure 3 presents the implemented solution, and the interaction between the subsystems.

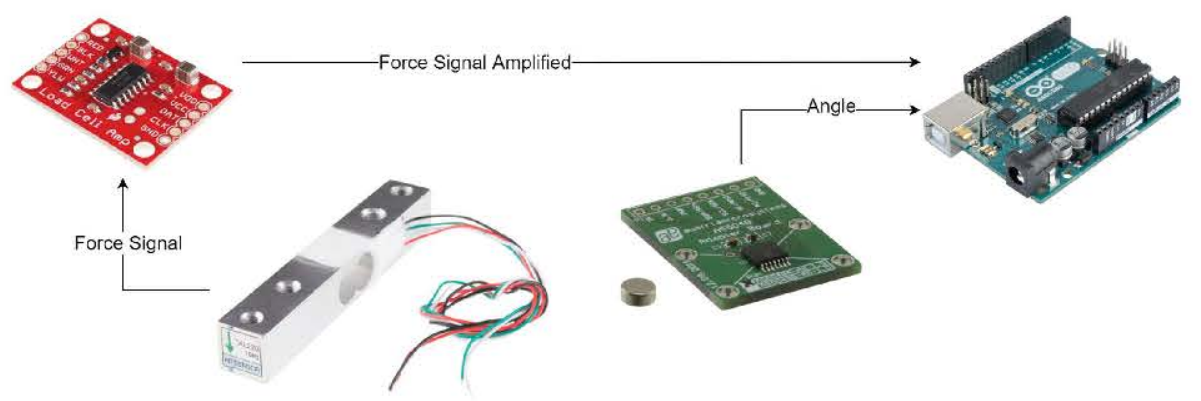

Fig. 3. Hardware diagram 


\section{Results}

In order to perform the hardware tests, a system with the components presented in Fig. 3 was built. This is presented in Fig. 4, and it was designed by the authors, being posteriorly $3 \mathrm{D}$ printed. The parts already provide space specially allocated for the load cell, as well as for its amplifier, and also for the absolute encoder. The most complicated task was to physically align the rotation axis of the joint, which has the magnet placed in its center, and the center of the Hall sensor. Taking into account the collected data, this task was considered to be performed successfully, since the angular displacement read by the sensor and by a physical angle meter were very similar.

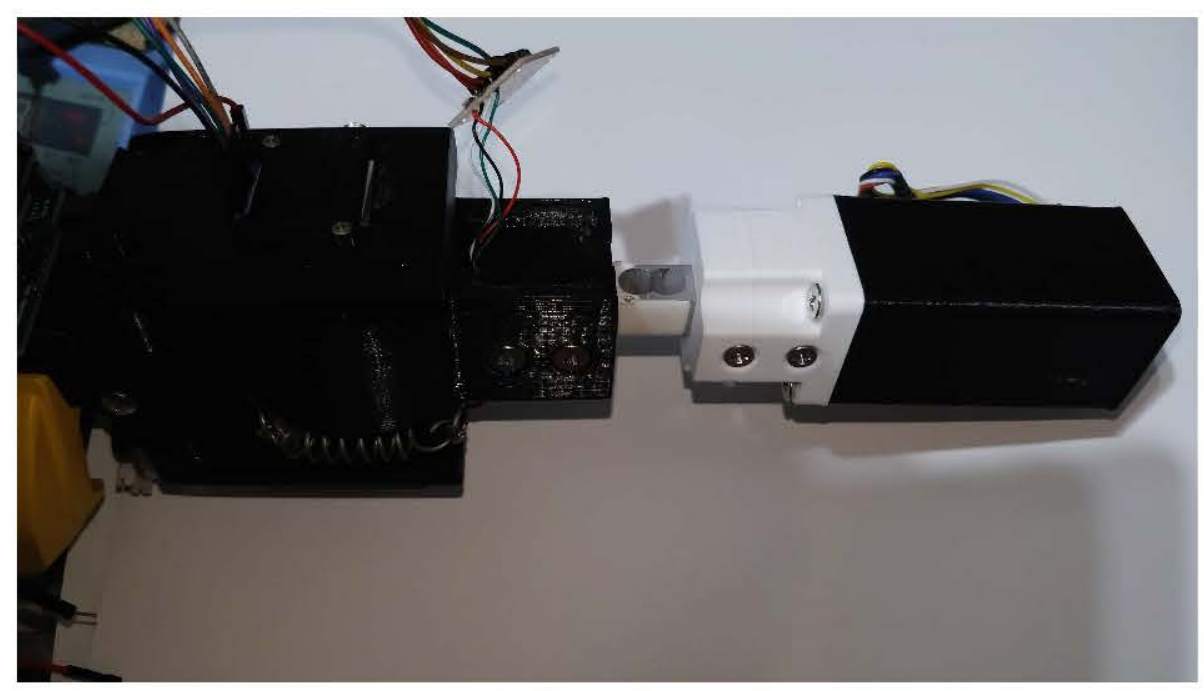

Fig. 4. Real joint

For the first set of tests, the system was acquiring data continuously, in order to recognize the variations in force exerted on the cell, with information of the angle. This test allows to know what is the behavior of the joint when its center of rotation is locked, and the posterior link is applying a force on it. This force is traduced to a torque, since it was put with some distance from the center. The results from this test are present graphically in Fig. 5. Since the joint is already coupled to a motor attached to a gearbox system, and since the gearbox present some backlash, the joint presents a dead zone. This behaviour is clearly visible in the figure, in the red line of the graph where, although the exerted force is practically zero, the angle has a significant variation. Considering that the motor is stopped, the difference between the angles of the motor shaft and the joint is obtained by the angle in the absolute encoder. So, using Eq. 1, and calculating the average of the straight slopes of the values from the positive and the negative torques, the value of $K$ is obtained and is equal to 7.3035. 


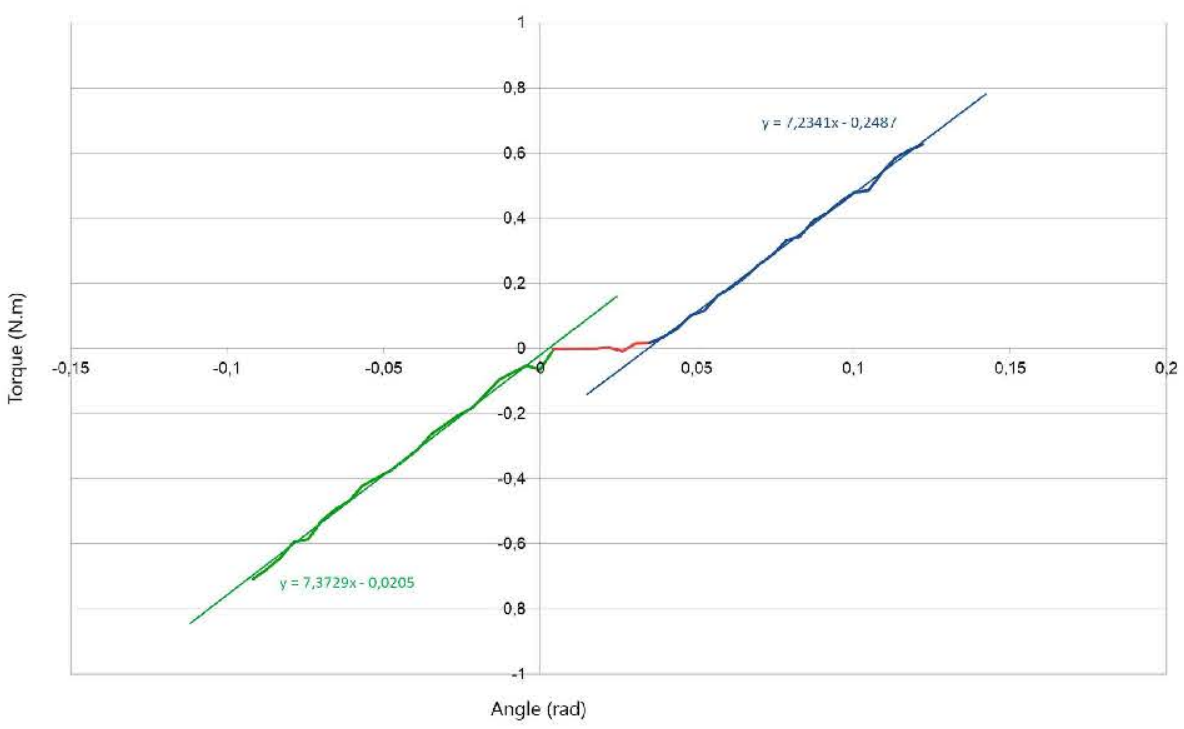

Fig. 5. Plot of Torque vs Angle

Then, to find the value of $J$ and $B$, it was necessary to proceed to a second set of tests. This consists of placing the system to acquire data, rotating the posterior link to a certain angle and releasing it, allowing its free oscillation until reaching the equilibrium point again. The results obtained are compiled in Table 2. Using Eq. 11, and the inverse Laplace transform, the following Equations could be used to represent the system in the time domain.

$$
\begin{gathered}
\theta(t)=\theta_{0} \cdot \sqrt{\frac{\left(\frac{\alpha}{\omega_{n}}-\xi \cdot \omega_{n}\right)^{2}}{1-\xi^{2}}+1} \cdot e^{-\xi \omega_{n} t} \sin \left(\omega_{n} \cdot \sqrt{1-\xi^{2}} \cdot t+\phi\right) \\
\phi=\tan ^{-1}\left(\frac{\omega_{n} \sqrt{1-\xi^{2}}}{\alpha-\xi \omega_{n}}\right)
\end{gathered}
$$

Having defined the approximation function, and since the value of $K$ was already estimated, the optimization process that minimizes the absolute error to the obtained angular position was put to run and the values of the variables for Eq. 12 were found. Establishing the parallelism between the function in the Laplace domain used and Eq. 11, after the optimization process, the values found for $B$ and $J$ were 0.0416 and 0.0085 . 
Considering a simple pendulum configuration, $J=m \cdot r^{2}$. If a weight of $269 \mathrm{gr}$. is put on a known point of the link, there is a known moment of inertia. Taking the value obtained for $J=0.0085$, and placing the known mass approximately $18 \mathrm{~cm}$ from the axis of rotation, one obtain an estimated value for the distance $r=17.78 \mathrm{~cm}$, an error of less than $1 \%$, which indicates that the estimation of the parameters associated with the joint has been carried out successfully. All the obtained estimated parameters are compiled in Table 1.

Table 1. Estimated joint parameters

\begin{tabular}{l|l}
\hline \multicolumn{2}{l}{ Parameters } \\
\hline $\mathrm{K}$ & $7.3035 \mathrm{~N} / \mathrm{m}$ \\
\hline B & $0.0416 \mathrm{~N} \cdot \mathrm{s} / \mathrm{m}$ \\
\hline $\mathrm{J}$ & $0.0085 \mathrm{Kg} \cdot \mathrm{m}^{2}$ \\
\hline
\end{tabular}

In Fig. 6, in blue there is the real measured behaviour of the joint, while in red there is the estimated model of the joint. Actually, approximately from $t=1 \mathrm{~s}$, it begins to be noticed that the model differs somewhat from the performed measurements. However, this difference can be explained by the non-linear behavior presented by the dead zone of the joint, which will have to be compensated by a suitable controller.

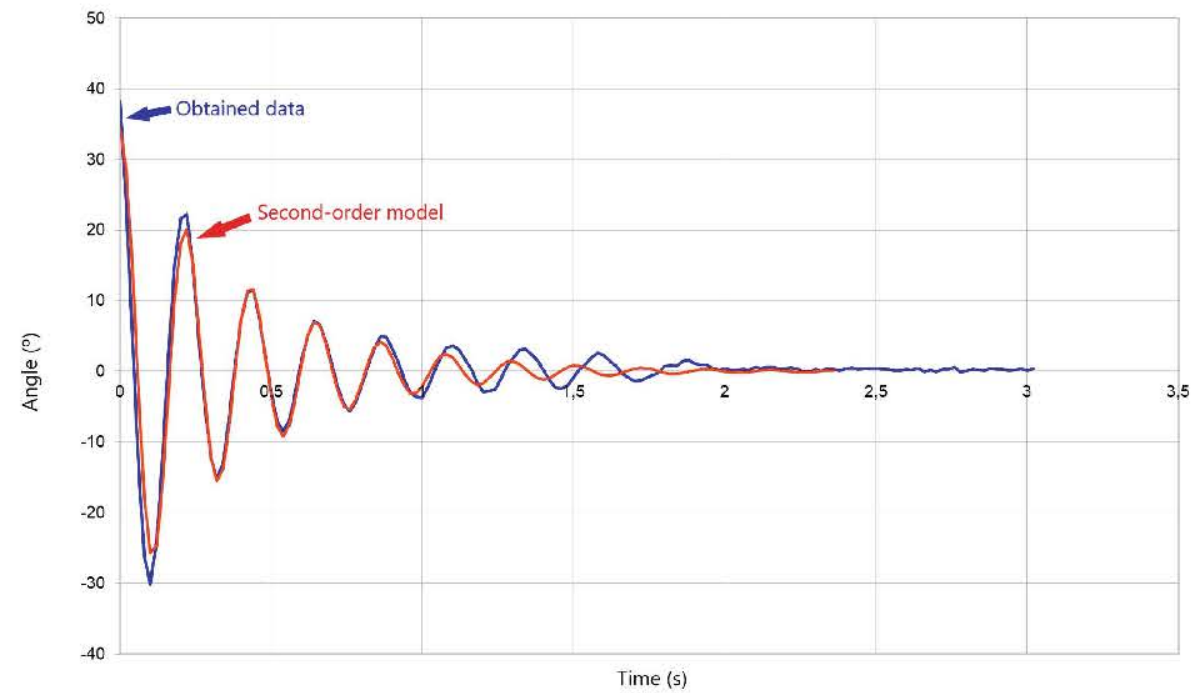

Fig. 6. Plot of Angle vs Time 
Table 2. Second-order model

\begin{tabular}{|c|c|c|c|}
\hline Time (s) & $\theta\left(^{\circ}\right)$ & $\theta_{\text {est }}\left({ }^{\circ}\right)$ & Error \\
\hline 0 & 38.14 & 34.28 & 3.85 \\
\hline 0.02 & 23.77 & 28.75 & 4.98 \\
\hline 0.04 & 4.42 & 14.61 & 10.19 \\
\hline 0.06 & -13.36 & -2.86 & 10.50 \\
\hline 0.08 & -26.17 & -17.79 & 8.38 \\
\hline 0.1 & -30.19 & -25.69 & 4.50 \\
\hline 0.12 & -24.04 & -24.70 & 0.66 \\
\hline 0.14 & -11.84 & -15.96 & 4.12 \\
\hline 0.16 & 2.37 & -2.97 & 5.34 \\
\hline 0.18 & 14.85 & 9.76 & 5.09 \\
\hline 0.2 & 21.6 & 18.21 & 3.39 \\
\hline 0.22 & 22.24 & 20.09 & 2.15 \\
\hline 0.24 & 15.42 & 15.42 & $1.24 \mathrm{E}-06$ \\
\hline 0.26 & 4.9 & 6.29 & 1.39 \\
\hline 0.28 & -4.94 & -3.99 & 0.95 \\
\hline 0.3 & -12.13 & -12.05 & 0.08 \\
\hline 0.32 & -15.36 & -15.53 & 0.17 \\
\hline 0.34 & -12.85 & -13.76 & 0.91 \\
\hline 0.36 & -6.61 & -7.79 & 1.18 \\
\hline 0.38 & 0.88 & 0.11 & 0.77 \\
\hline 0.4 & 7.23 & 7.23 & $1.18 \mathrm{E}-08$ \\
\hline 0.42 & 11.1 & 11.40 & 0.30 \\
\hline 0.44 & 11.56 & 11.56 & $1.46 \mathrm{E}-07$ \\
\hline 0.46 & 7.65 & 8.04 & 0.39 \\
\hline 0.48 & 2.37 & 2.29 & 0.08 \\
\hline 0.5 & -3.19 & -3.65 & 0.46 \\
\hline 0.52 & -7.23 & -7.88 & 0.65 \\
\hline 0.54 & -8.48 & -9.21 & 0.73 \\
\hline 0.56 & -6.79 & -7.50 & 0.71 \\
\hline 0.58 & -2.59 & -3.57 & 0.98 \\
\hline 0.6 & 1.65 & 1.14 & 0.51 \\
\hline 0.62 & 4.92 & 5.04 & 0.12 \\
\hline 0.64 & 7.12 & 6.98 & 0.14 \\
\hline 0.66 & 6.64 & 6.53 & 0.11 \\
\hline 0.68 & 4.28 & 4.04 & 0.24 \\
\hline
\end{tabular}




\section{Conclusions and Future Work}

As a first conclusion, the physical implementation of the idealized and simulated prototype of a novel elastic joint have met the expectations, adding a damping component to the joint. The addition of the 4 springs allowed the joint to have an elastic behavior with only passive components, and consequently without energy consumption.

Then, it can be concluded that, based on a purely low-cost sensor acquisition system, it is possible to carry out tests and consider measurements that allow to estimate parameters of a complex model. Regarding the model, the estimated parameters were used to compare measurements with real values and have a very low error, so this process can be considered as successfully achieved.

Finally, in the overlap between the function in order of time of the model developed and the measures that were obtained from the real system, differences are effectively noticed, which come from the joint's dead zone. These differences will therefore have to be compensated in the design of a suitable controller.

Therefore, the future work is to design a suitable closed-loop controller that allows to control the joint in position, and that tries to reduce the non-linear effects that the dead zone adds to the model.

Acknowledgements. This work is financed by National Funds through the Portuguese funding agency, FCT - Fundação para a Ciência e a Tecnologia, within project UIDB/50014/2020.

\section{References}

1. Liu, H., Cui, S., Liu, Y., Ren, Y., Sun, Y.: Design and vibration suppression control of a modular elastic joint. Sensors 18(6), 1869 (2018)

2. Giusti, A., Malzahn, J., Tsagarakis, N.G., Althoff, M.: On the combined inversedynamics/passivity-based control of elastic-joint robots. IEEE Trans. Rob. 34(6), 1461-1471 (2018)

3. Okui, M., Iikawa, S., Yamada, Y., Nakamura, T.: Variable viscoelastic joint system and its application to exoskeleton. In: 2017 IEEE/RSJ International Conference on Intelligent Robots and Systems (IROS), pp. 3897-3902. IEEE (2017)

4. Zhang, T., Huang, H., Guo, H., Li, B.: Singularity avoidance for a deployable mechanism using elastic joints. J. Mech. Des. 141(9), 094501 (2019)

5. Kau, N., Schultz, A., Ferrante, N., Slade, P.: Stanford doggo: an open-source, quasi-direct-drive quadruped. In: 2019 International Conference on Robotics and Automation (ICRA), pp. 6309 6315. IEEE (2019)

6. Montazeri, A., West, C., Monk, S.D., Taylor, C.J.: Dynamic modelling and parameter estimation of a hydraulic robot manipulator using a multi-objective genetic algorithm. Int. J. Control 90(4), 661-683 (2017)

7. González, A., Cerda-Lugo, A., Cardenas, A., Maya, M., Piovesan, D.: A third-order model of hip and ankle joints during balance recovery: modeling and parameter estimation. J. Comput. Nonlinear Dyn. 14(10), 101001 (2019)

8. Ge, W., Wang, B., Mu, H.: Dynamic parameter identification for reconfigurable robot using adaline neural network. In: 2019 IEEE International Conference on Mechatronics and Automation (ICMA), pp. 319-324. IEEE (2019) 
9. Miranda-Colorado, R., Moreno-Valenzuela, J.: Experimental parameter identification of flexible joint robot manipulators. Robotica 36(3), 313-332 (2018)

10. Ni, H., Zhang, C., Hu, T., Wang, T., Chen, Q., Chen, C.: A dynamic parameter identification method of industrial robots considering joint elasticity. Int. J. Adv. Rob. Syst. 16(1), 1729881418825217 (2019) 